\title{
Measuring suspended sand transport using a pulse-coherent acoustic Doppler profiler
}

\author{
T. Aagaard ${ }^{1} \&$ B. Greenwood ${ }^{2}$ \\ ${ }^{1}$ Institute of Geography and Geology, University of Copenhagen, \\ Denmark \\ ${ }^{2}$ Department of Physical and Environmental Sciences, \\ University of Toronto at Scarborough, Canada
}

\begin{abstract}
A simple method to derive sediment transport rates in the nearshore from PulseCoherent Acoustic Doppler Profilers (PC-ADP) is described. Measured sediment concentration profiles are compared with theoretically estimated concentration profiles and exhibit good agreement. Cross-shore and longshore sediment transport rates obtained during 5-6 week long deployments on the lower shoreface in 6-8 $\mathrm{m}$ water depth are compared with predictions from an energetics transport model. The two estimates agree to within $20 \%$ in the longshore direction while the cumulated cross-shore wave-dominated transport component is trending significantly less offshore for the measurements than for the model predictions. The measurements indicate that significant onshore sediment transport from the lower to the upper shoreface only occurs when waves are close to breaking.
\end{abstract}

Keywords: PC-ADP, sediment concentrations, sediment transport, shoreface.

\section{Introduction}

Field measurements of suspended sediment transport in the nearshore can be obtained using either optic or acoustic devices (Osborne et al. [1]). While standard procedures exist for estimating sediment concentrations from optical backscatter, it is considerably more difficult to obtain reliable estimates from acoustic backscatter sensors (ABS). One reason is that acoustic backscatter needs to be corrected for signal attenuation due to water absorption and sediment scattering. Significant effort has been invested in developing algorithms to invert 
the backscattered acoustic signal to provide sediment concentration (e.g. Betteridge et al. [2]; Sheng and Hay [3]; Thorne and Hanes [4]) but while reliable results from ABS can now be obtained, procedures for signal correction are complicated and time-consuming when long-term sediment transport rates need to be assessed. In addition, unless multi-frequency sensors are used (e.g. Crawford and Hay [5]), these techniques can only be used for single grain sizes and are difficult to apply for sand when water clarity is significantly affected by for example more or less permanently suspended silts and/or organics.

Here, we test a simple method for obtaining long-term sediment transport rates from a Pulse-Coherent Acoustic Doppler Profiler (PC-ADP). The PC-ADP is similar to ABS sensors in the sense that backscattered sound pressure is proportional to the amount of scatterers (sediment particles) in the water column. In contrast to ABS, PC-ADP's have only rarely been used to study sediment dynamics (Kostachuk et al. [6]; Wren and Leonard [7]), possibly because of the relatively large footprint of the sensor (approx. $0.13 \mathrm{~m}$ at a distance of $0.50 \mathrm{~m}$ above the bed). Hence, while the reliability of velocity estimates from PC-ADP's have been established recently (Lacy and Sherwood [8]), the capability of the instrument to resolve sediment transport is not well understood.

However, when estimating sediment transport in the bottom boundary layer under waves, it is important that velocity and sediment concentration measurements are collocated. This is the case for PC-ADP sensors because velocity and backscatter is recorded at the same point in the water column, while ABS and paired velocity sensors are separated by some finite distance at least in the horizontal. In addition, the large sensor footprint of the PC-ADP may be an advantage in some respects because the effect of bedforms on sediment suspension and concentration will be averaged over an entire bedform. In this paper, mean sediment concentration profiles and concentration time series estimated using a simple methodology are compared to outputs determined from more elaborate techniques. Cross-shore and longshore sediment transport rates are then compared with predictions from an often-used energetics sediment transport model to assess sediment transport rates on the lower shoreface under a range of wave conditions.

\section{Study site}

The field site at Vejers Beach is located on the exposed Danish North Sea coast. The mean annual offshore significant wave height $\left(H_{s}\right)$ is $1.3 \mathrm{~m}$ with an average zero-crossing wave period $\left(T_{z}\right)$ of $4.3 \mathrm{~s}$. Waves from the northwest are dominant and offshore significant wave heights are up to $7 \mathrm{~m}$ during storms. Persistent low $\left(\mathrm{H}_{\mathrm{s}}<1 \mathrm{~m}\right)$ background swell impinges from the northwest. The site is microtidal with a mean spring tidal range of $1.2 \mathrm{~m}$. Tidal current speeds on the lower shoreface are in the order of $0.2-0.3 \mathrm{~m} \mathrm{~s}^{-1}$ with a northward directed residual current.

The cross-shore profile and the mean sand grain size at the study site are illustrated in Figure 1. The shoreface at Vejers has a mean slope of $\beta=0.006$ and it exhibits 3-4 longshore bars. The PC-ADP was deployed seaward of the bars in 
mean water depths of 6 and $8 \mathrm{~m}$, respectively, for periods of 5-6 weeks during the fall seasons of 2005 and 2006. The shallower deployment depth corresponds to the boundary between the upper and the lower shoreface where bathymetric change over time pinches out, see Figure 2 . The mean sand grain size on the lower shoreface is $\approx 120$ microns and it coarsens to $\approx 180$ microns on the upper shoreface (Figure 1). In addition, significant amounts of permanently suspended silts and organics (wash load) are present in the area; this sediment originates from the Wadden Sea region further south and is transported northward by the residual tidal currents.

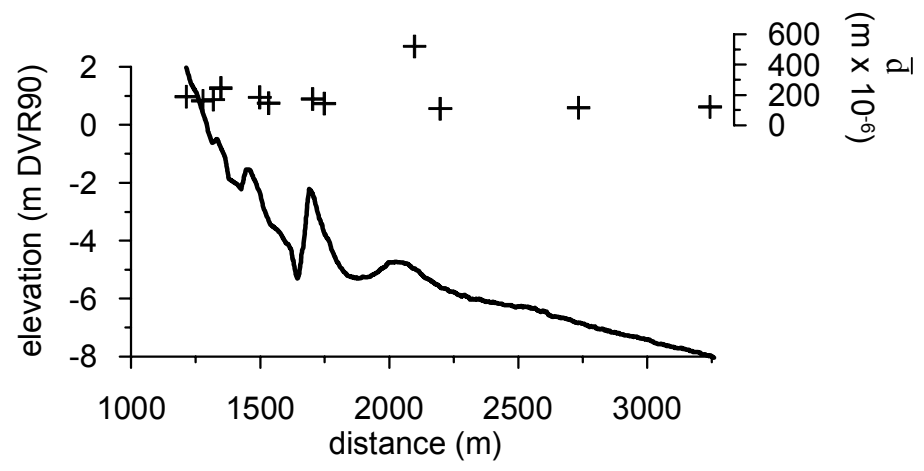

Figure 1: The cross-shore bathymetry of the field site at Vejers at the time of the first instrument deployment. The right-hand axis shows mean sediment grain size across the profile. DVR90 is Danish Ordnance datum, corresponding to mean sea level.

\section{Methodology}

At both deployment positions, a $1.5 \mathrm{MHz}$ Sontek PC-ADP was mounted $0.51 \mathrm{~m}$ above the bed and recorded fluid velocities and acoustic backscatter intensity in $1.6 \mathrm{~cm}$ vertical bins from the sensor blanking distance $(0.05 \mathrm{~m}$ from the transducer head) to the seabed. The sampling mode was one burst per hour with a record duration of 8.5 minutes and a sampling rate of $2 \mathrm{~Hz}$. A Druck pressure sensor was installed in the transducer head to record wave heights and mean water depths. Velocity outputs were rotated with respect to shoreline orientation to provide cross-shore $(u)$ and alongshore $(v)$ velocities, they were corrected for ambient sound speed using data from a CTD-probe, smoothed using a horizontal 3-point Box filter and velocity ambiguity resolution was applied when required. When true velocities exceed the maximum velocity resolvable by the instrument, the velocity signal is wrapped resulting in erroneous readings. A second set of acoustic pulse pairs allows the signal to become unwrapped. For the particular instrument and the given profiling lag, velocity ambiguities occurred when orbital velocity (predicted from the local wave height measurements) exceeded $0.65 \mathrm{~m} \mathrm{~s}^{-1}$ near the bed. Records containing velocities theoretically exceeding 
this threshold were corrected using the software supplied by the manufacturer and subsequently despiked and smoothed.

Acoustic backscatter intensity was used to estimate suspended sediment concentrations $(c)$ and subsequently cross-shore $\left(q_{x}\right)$ and longshore $\left(q_{y}\right)$ transport rates. Methods to compute $c$ directly from backscattered pressure, accounting for signal loss due to water absorption and sediment scattering (e.g. Betteridge et al. [2]; Thorne and Hanes [4]; Crawford and Hay [5]) are difficult to apply for single-frequency sensors when the sediment is inhomogeneous, consisting of a mixture of sand and wash load, and they usually further require an independent estimate of $c$ at some point within the water column. The latter could not be achieved for the deployments at Vejers because the tripod settled some $15 \mathrm{~cm}$ into the bed upon deployment and an OBS-3 optical backscatter sensor mounted at a nominal elevation of $0.05 \mathrm{~m}$ above the bed was below bed level, or interfering with the bed during the deployment periods.

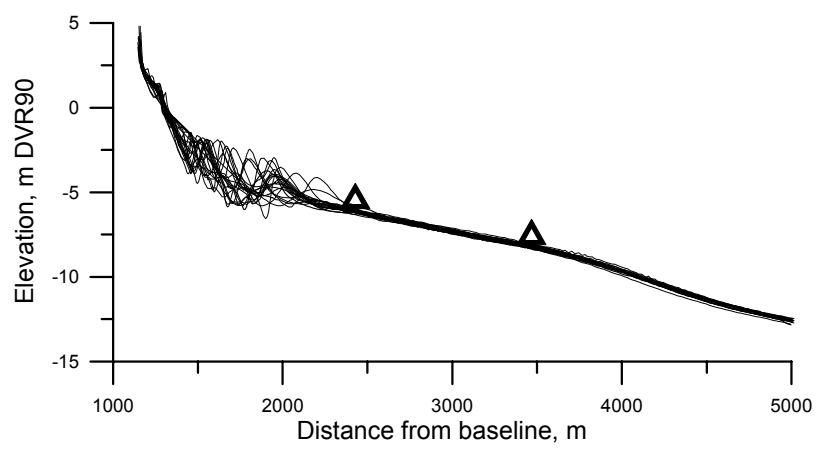

Figure 2: Cross-shore profile envelope comprising surveys 1969-2006 at Line 6170 off Vejers. The two triangles indicate the PC-ADP deployment positions.

Instead, a simple method of obtaining the concentration of sand in the water was developed: Acoustic backscatter was corrected for signal loss due to sound attenuation by water absorption and by the permanently suspended wash load. This attenuation was determined from instrument bursts with negligible wave activity and a lack of sediment suspension from the bed, and it was estimated as $-18 \mathrm{~dB} \mathrm{~m}^{-1}$. Field offsets induced by inherent system characteristics and wash load were then removed from the individual instrument records. They were taken as the average over the uppermost five measurement bins of the $5^{\text {th }}$ percentile of output signal values and subtracted from the signals in all bins assuming that the fine-grained sediment fractions were uniformly distributed in the vertical. Acoustic pressure was then converted to sediment concentration using a transfer function that was developed from a short-term experiment during which measurements of optical backscatter were available. This experiment was done at a location with comparable sand characteristics $(\bar{d}=148$ microns $)$ and the OBSsensor was subsequently calibrated in a recirculating tank to obtain a crosscalibration between backscattered acoustic pressure and sediment concentration. 
The resulting transfer function was applied to all acoustic bins after offset removal. Hence, backscatter intensity was not corrected for sound attenuation due to sediment scattering. However, failure to do so may not a large problem, as reported errors are typically within $\pm 20 \%$ when the sensor operating frequency is less than $3 \mathrm{MHz}$ (Vincent [9]).

Finally, net cross-shore and longshore suspended sediment transport rates were estimated using:

$$
q=\sum_{n=1}^{N} \sum_{t=0}^{T} u(t) c(t) d n d t
$$

where $u$ is fluid velocity, $c$ is (estimated) sediment concentration, $N$ is the number of measurement bins $(N=22-26)$ and $T$ is the duration of the time series.

\section{Mean concentration profiles}

To compare the predictions of $c$ from the simple method with estimates using a standard algorithm and to assess the quality of the predicted sediment concentrations, measurements from the short-term deployment were used. In this case, an OBS-sensor was located at an elevation of $8-10 \mathrm{~cm}$ above the bed. The instruments were deployed in a trough landward of a longshore bar, and the seabed was covered with wave ripples having a wavelength of $11 \mathrm{~cm}$ and a height of $2 \mathrm{~cm}$. The significant wave height for the particular example shown here was $0.33 \mathrm{~m}$ with a period of $5.6 \mathrm{~s}$ and the mean water depth was $1.31 \mathrm{~m}$ yielding a relative wave height of 0.25 ; the waves were non-breaking.

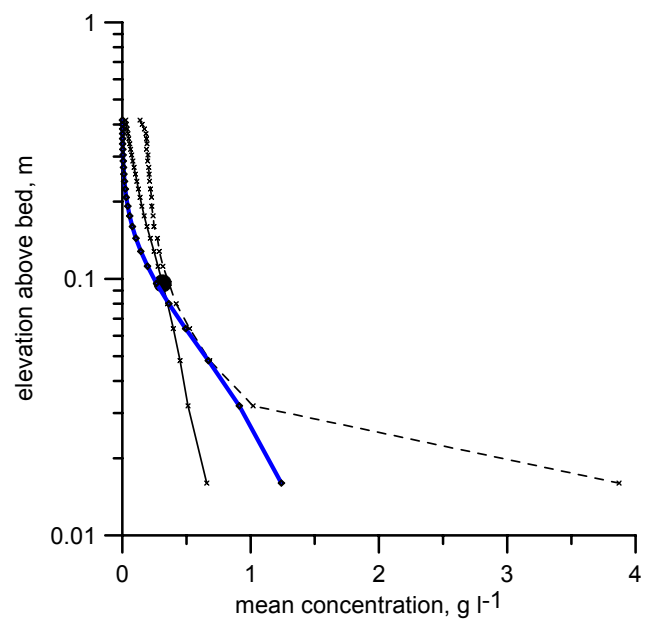

Figure 3: $\quad$ Mean concentration profiles determined from the simple method (dashed line) and from the explicit method of Thorne and Hanes [4; solid line]. The dot indicates the mean concentration recorded by the OBS. The thick blue line is the mean concentration profile determined from eqn. (2). 
The mean concentration profile was determined from the simple method outlined above and from the explicit method of Lee and Hanes [10] and Thorne and Hanes [4; their eqn.21]. The explicit method requires an independent estimate of the sediment concentration at a given elevation, which is obtained from the OBS and it assumes a constant particle diameter with elevation above the bed. The attenuation due to water was taken as $0.067 \mathrm{~N} \mathrm{~m}^{-1}$ (Thorne et al. [11]) and the sediment attenuation was computed from Thorne and Hanes [4; their eqn.9]. The output values were finally corrected for near-field effects. Comparing the two estimates (Figure 3), the mean concentration profile obtained from the explicit method is considerably steeper, while measured concentrations increase more rapidly towards the bed.

The results were also compared with the independent model of Nielsen [12]:

$$
C(z)=C_{0} \exp \left(-\frac{z}{l_{s}}\right)
$$

where $C_{0}$ is a reference concentration near the bed, given as $C_{0}=0.005 \theta_{r}^{3}$ with $\theta_{r}$ being the effective Shields parameter in the presence of ripples (Nielsen [13]). $z$ is elevation above the bed, and the vertical mixing length, $l_{s}$, is computed from Aagaard [14]:

$$
\frac{l_{s}}{h}=0.012 \exp \left(4.78 \frac{H_{s}}{h}\right)
$$

Except for the lowermost measurement point which may be affected by proximity to the bed (with the sensor in fact intermittently seeing the bed), the concentrations determined from the simple method are much closer to the independent model predictions than the explicit method, probably because the explicit method assumes a homogeneous sand suspension and does not account for signal dampening by the wash load.

\section{Instantaneous sediment concentrations}

Instantaneous values of sediment concentration, $c(t)$, are required in order to calculate sediment transport rates in wave-dominated settings and $c(t)$ can not be obtained from the explicit method which considers only mean concentrations. Instantaneous sediment concentrations obtained from the simple method were instead compared with the time series from the OBS, and with predictions using the algorithm provided by Osborne et al. [1; their eqn.1]; the required system calibration was obtained from the method of Betteridge et al. [2] and attenuation due to sediment scattering was determined from the high-pass model of Sheng and Hay [3]).

Figure 4 compares the three sediment concentration time series at an elevation of $9.6 \mathrm{~cm}$ above the bed. The measured time series from the PC-ADP captures the basic characteristics of the concentration time series as seen by the OBS, although the two time series signals are not identical. The two instruments were separated in the horizontal by approximately $0.5 \mathrm{~m}$ and the measurement volume of the ADP is far larger than the measurement volume of the OBS. The latter is 
conceptually a point measurement of sediment concentrations, while the former provides an estimate which is averaged over approximately $80 \mathrm{~cm}^{2}$, at this particular distance from the sensor head. Hence, the diameter of the sensing volume roughly corresponded to the ripple wavelength and the concentration estimate from the ADP is a measure of the average concentration over one ripple form.

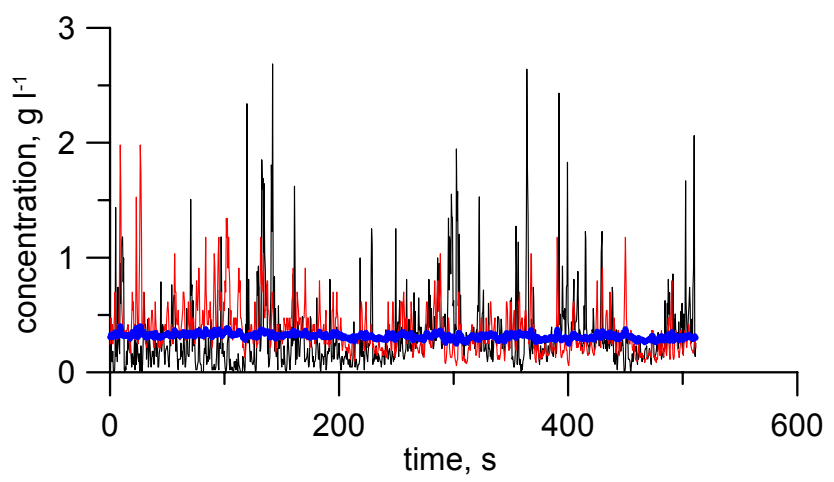

Figure 4: $\quad$ Time series of sediment concentrations at $9.6 \mathrm{~cm}$ above the bed measured by the OBS (black), by the PC-ADP (red) and estimated from the method of Osborne et al. [1; thick blue line].

In contrast to the time series from the ADP, the time series predicted from the method of Osborne et al. [1] bears little resemblance to the actually occurring concentrations and completely lacks the spiky nature of the observed concentration signal. The reason is probably again that the wash load dampens the amplitude of the backscattered signal and this effect is not taken into account by the model. In contrast, the simple method first removes the offset induced by the fine-grained sediment and then uses the cross-correlation with the OBSsignal from which the offset has also been removed.

\section{Net sediment transport}

The acoustic backscatter calibration obtained from the trial experiment was applied to estimate sediment concentrations and transports during the experiments at Vejers. Significant wave height, mean current speeds and longshore and cross-shore instantaneous and cumulated sediment transport from the experiment at $6 \mathrm{~m}$ water depth are plotted in Figure 5. The figure also plots suspended sediment transports rates estimated from the Bailard energetics model:

$$
<q_{t}(t)>=K_{s}\left(<|\vec{u}|^{3}(t) u(t)>\right)-K_{s g}<|\vec{u}|^{5}(t)>
$$



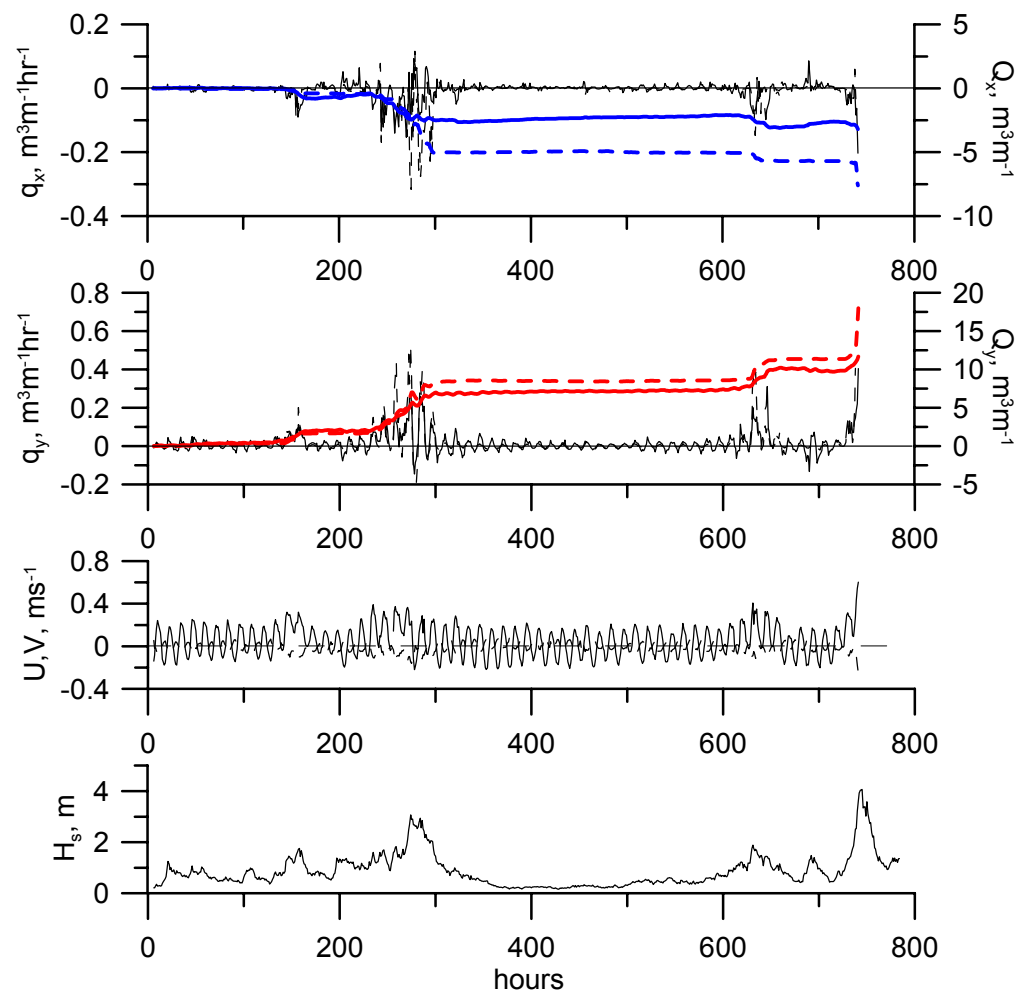

Figure 5: $\quad$ Measurements from the PC-ADP deployment in $6 \mathrm{~m}$ water depth at Vejers. The lower panel shows significant wave height $\left(H_{s}\right)$ and the second panel shows cross-shore ( $U$; dashed) and longshore $(V$; solid) mean current speeds. The third panel from the bottom illustrates measured (solid line) and model-predicted (dashed line) suspended longshore sediment transport rates $\left(q_{y}\right)$ and the upper panel shows the cross-shore transport rates $\left(q_{x}\right)$. The thick coloured lines illustrate the cumulated transport rates with the solid lines representing measurements and dashed lines representing model predictions. Positive transports are directed onshore and northward.

where the suspended load term is:

$$
K_{s}=\left(\frac{\rho}{\rho_{s}-\rho}\right) c_{f} g a^{\prime}\left(\frac{e_{s}}{w_{s}}\right)
$$

and the gravity term is

$$
K_{s g}=K_{s}\left(\frac{e_{s}}{w_{s}}\right) \tan \beta
$$


In eqn. (4), $u(t)$ is the instantaneous cross-shore velocity, $\vec{u}(t)$ is the magnitude of the instantaneous horizontal velocity vector, $\rho_{s}$ and $\rho$ are sediment and water density, $g$ is gravitational acceleration, $a^{\prime}$ is the pore space factor (0.6), $\tan \beta$ is the local slope of the bed, $\tan \varphi$ is angle of sediment repose, $w_{s}$ is sediment fall velocity, taken as $0.012 \mathrm{~m} \mathrm{~s}^{-1}$, and the transport efficiency factors were set at the model default values $e_{b}=0.21$ and $e_{s}=0.025$ (Bailard [15]). $c_{f}$ is a drag coefficient $=f_{w} / 2$, where $f_{w}$ is the wave friction factor. The longshore transport component was calculated accordingly. Velocities recorded at $0.25 \mathrm{~m}$ above the bed were used as input to the model

In the longshore direction, measured cumulated longshore sediment transport agrees with model predictions to within $20 \%$, and time-dependent transport rates were in good agreement, except for situations when wave heights exceeded $3 \mathrm{~m}$. In such cases, the energetics model provides larger transport rates which may be because the measurements were not corrected for sound attenuation due to sediment scattering; this is considered to be more significant when waves (and therefore sediment concentrations) are large.

In the cross-shore direction where sediment transport due to wave motions is much more important, measurements trended significantly more onshore than model predictions. While the energetics model can be expected to perform well when mean currents are dominant, which was the case for the longshore transport, it generally provides imprecise results when wave motions are dominant (Thornton et al. [16]). Reasons for the model inaccuracies include the assumption of in-phase relationships between velocity and concentration. In reality, phase shifts between velocity and concentration tend to develop when bedforms are present. For the present measurements, cumulated (offshoredirected) model-predicted transport was a factor of 2 larger than the measured transports, and the model does not replicate the onshore transport that is indicated by the measurements during low- and moderate energy situations (Figure 5; hours 350-600 and 650-700).

\section{Sediment supply from the lower to the upper shoreface}

The supply of sand from the lower to the upper shoreface is an important factor to the long-term development of the cross-shore profile. Figure 6 plots the measured cross-shore sediment transport rates obtained at 6 and $8 \mathrm{~m}$ depth as a function of relative wave height and the mobility number:

$$
\psi=\frac{\vec{u}^{2}}{(s-1) g \bar{d}}
$$

where $(s-1)$ is relative sediment density and $\bar{d}$ is mean sediment grain size. According to Dingler and Inman [17], ripples occur for $\psi<240$, while flat bed and sheet flow prevail for $\psi>240$.

Because tidal currents set obliquely to the depth contours at Vejers, the mean currents introduce a site-specific bias which is not representative in a general assessment of cross-shore sediment exchange between the upper and lower 
shoreface. Therefore, only the wave-induced oscillatory transport rates are considered in Figure 6 where mean (current-induced) transports rates have been subtracted from the total transport rate. Oscillatory transports were mainly onshore directed while the total net transports at $6 \mathrm{~m}$ depth (Figure 5) were mainly offshore directed, because of the dominance of the mean transport component at this depth at Vejers.
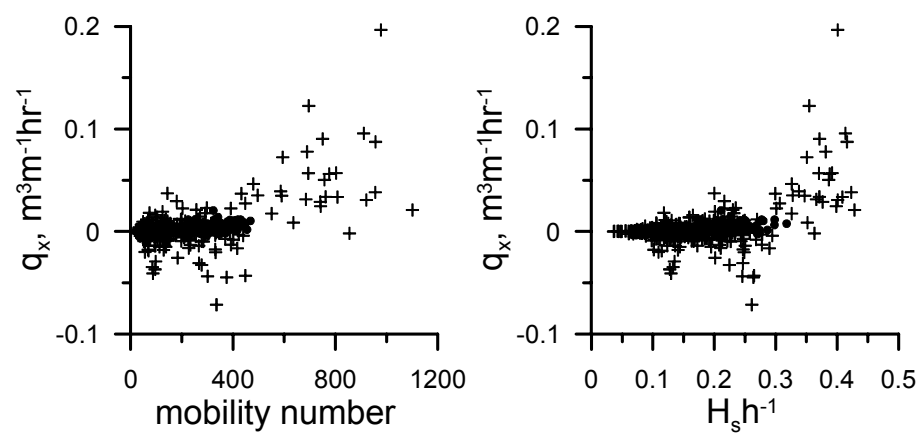

Figure 6: Net wave-induced cross-shore suspended sediment transport rates at $6 \mathrm{~m}$ (crosses) and $8 \mathrm{~m}$ (dots) water depth, as a function of mobility number and relative wave height. Positive transport rates are directed onshore.

The measurements suggest that significant onshore transport rates due to waves on the lower shoreface at occur only when $\psi>\approx 400$ and the relative wave height $H_{s} / h>\approx 0.3$. This corresponds to flat bed conditions when waves are strongly shoaling and close to breaking. For smaller relative wave heights and when bedforms occur, net cross-shore wave-induced transport rates were small and inconsistent at Vejers. This indicates that sediment supply from the lower to the upper shoreface may be limited and occurs from a rather narrow zone seaward of the longshore bars.

\section{Conclusions}

Acoustic backscatter sensors are increasingly used to estimate sediment concentrations and transports on the shoreface, and algorithms exist for inversion of the backscattered signal to provide sediment concentrations. Due to their complexity, these algorithms may not be favourable for using with large data sets and they normally assume uniform sediment grain sizes. Here, we have derived a simple method to obtain sediment concentrations and transport rates from PCADP sensors in settings with a coexistence of sand and wash load. The method is based on a transfer function between acoustic backscatter and concentration, and using a heuristic estimate of sound attenuation caused by water absorption and wash load. Tests suggest that this method performs better than accepted methods under the particular conditions sampled. Data comparison with predictions from a frequently used sediment transport model suggest that a PC-ADP can produce 
reasonable estimates of sediment transport rates on the shoreface, even when integrated over relatively long time scales. The results obtained further indicate that onshore transport rates from the lower to the upper shoreface are insignificant, unless waves are close to breaking.

\section{References}

[1] Osborne, P.D., Vincent, C.E. \& Greenwood, B. Measurement of suspended sand concentrations in the nearshore: field intercomparison of optical and acoustic backscatter sensors. Continental Shelf Research, 14, pp. 159-174, 1994.

[2] Betteridge, K.F.E., Thorne, P.D. \& Cooke, R.D. Calibrating multi frequency acoustic backscatter systems for studying near-bed suspended sediment transport processes. Continental Shelf Research, 28, pp. 227-235, 2008.

[3] Sheng, J. \& Hay, A.E. An examination of the spherical scatterer approximation in aqueous suspension of sand. Journal Acoustic Society of America, 83, pp. 598-610, 1988.

[4] Thorne, P.D. \& Hanes, D.M. A review of acoustic measurement of smallscale sediment processes. Continental Shelf Research, 22, pp. 603-632, 2002.

[5] Crawford, A.M. \& Hay, A.E. Determining suspended sand size and concentration from multifrequency acoustic backscatter. Journal Acoustic Society of America, 94, pp. 3312-3324, 1993.

[6] Kostachuk, R., Best, J., Villard, P., Peakall, J. \& Franklin, M. Measuring flow velocity and sediment transport with an acoustic Doppler current profiler. Geomorphology, 68, pp. 25-37, 2005.

[7] Wren, P.A. \& Leonard, L.A. Sediment transport on the mid-continental shelf, Onslow Bay, North Carolina during Hurricane Isabel. Estuarine, Coastal and Shelf Science, 63, pp. 43-56, 2005.

[8] Lacy, J.R. \& Sherwood, C.R. Accuracy of a pulse-coherent acoustic Doppler profiler in a wave-dominated flow. Journal Atmospheric and Oceanic Technology, 21, pp. 1448-1461, 2004.

[9] Vincent, C.E. Measuring suspended sand concentration using acoustic backscatter: a critical look at the errors and uncertainties. Coastal and Shelf Sediment Transport, ed. Balson, P.E. \& Collins, M.B., Geological Society of London, Special Publication 274, pp. 7-15, 2007.

[10] Lee, T.H. \& Hanes, D.M. Direct inversion method to measure the concentration profile of suspended particles using backscattered sound. Journal of Geophysical Research, 100, pp. 2649-2657, 1995.

[11] Thorne, P.D., Vincent, C.E., Hardcastle, P.J., Rehman, S. \& Pearson, N. Measuring suspended sediment concentrations using acoustic backscatter devices. Marine Geology, 98, pp. 7-16, 1991.

[12] Nielsen, P. Coastal Bottom Boundary Layers and Sediment Transport. World Scientific, Singapore, 324 pp, 1992. 
[13] Nielsen, P. Suspended sediment concentration under waves. Coastal Engineering, 10, pp. 23-31, 1986.

[14] Aagaard, T. Beach Morphodynamics: two Danish case studies. Medd. Skalling-Laboratoriet, 38, 160 pp, 2002.

[15] Bailard, J.A. An energetics total load sediment transport model for a plane sloping beach. Journal of Geophysical Research, 86, pp.10938-10954, 1981.

[16] Thornton, E.B., Humiston, R.T. \& Birkemeier, W.A. Bar/through generation on a natural beach. Journal of Geophysical Research, 101, pp. 12097-12110, 1996.

[17] Dingler, J.R. \& Inman, D.L. Wave-formed ripples in nearshore sands. Proc. $15^{\text {th }}$ Conf. on Coastal Eng., ASCE, New York, pp. 2109-2126, 1977. 